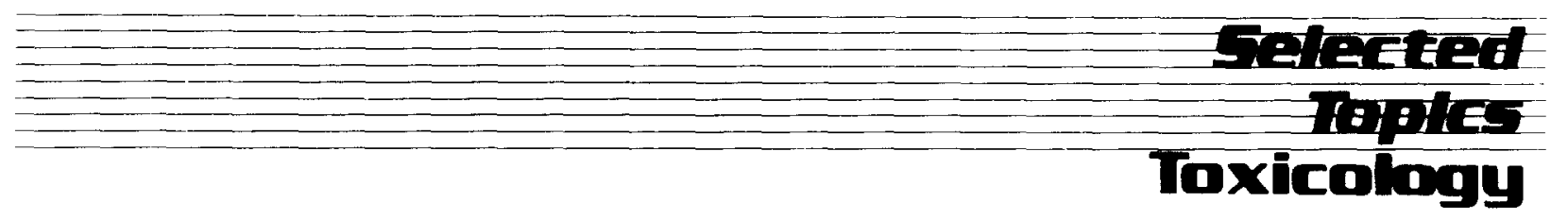

\title{
CRITERIA FOR ADMITTING PATIENTS WITH TRICYCLIC ANTIDEPRESSANT OVERDOSE
}

\author{
Glenn F. Tokarski, MD, and Mark J. Young, MD \\ Divisıon of General Medicine, Department of Internal Medıcıne, University of Michigan Medical Center, Ann Arbor \\ Reprint address: Mark J. Young, MD, Director of General Medicine, Department of Internal Medicine, Medical College of Wisconsin. \\ 8700 W. Wisconsin Ave., Milwaukee, WI 53226
}

\begin{abstract}
$\square$ Abstract - Several investigators have recently developed guidelines for determining which patients with tricyclic antidepressant overdose should be hospitalized. The width of the QRS complex on the ECG and several clinical parameters have been proposed to identify patients at risk for major complications. To validate these, we developed an algorithm and then applied it to 45 patients who had overdosed on tricyclic antidepressants. This algorithm correctly predicted which patients required admission, whether due to present or impending complications, and which patients could have been discharged without morbidity or mortality. We conclude that use of the modified algorithm can identify patients with tricyclic antidepressant overdose who can be safely discharged from the emergency department.
\end{abstract}

Keywords - algorithm; tricyclic; antidepressant; overdose; complications

\section{INTRODUCTION}

Tricyclic antidepressant (TCA) overdose may cause life-threatening complications. Most patients with TCA overdose are admitted to an intensive care unit because criteria for identifying patients who will have an uncomplicated course after TCA overdose have not been available..$^{1-6}$

Two investigators have attempted to predict which patients are at high risk. Callaham developed an algorithm based on clinical and ECG criteria, that identifies patients who are at high risk of major complications after TCA overdose. ${ }^{7}$ Boehnert and Lovejoy reported that the QRS duration is a useful predictor of seizures and ventricular dysrhythmias after TCA overdose. ${ }^{8}$ We combined these guidelines into a single algorithm and tested them on all patients who were evaluated for TCA overdose at the University of
Michigan Medical Center between 1982 and 1985. The specific objective of this study was to determine if the modified algorithm would retrospectively identify patients with both complicated and uncomplicated TCA overdose.

\section{METHODS}

We reviewed the care of all patients with TCA overdose at the University of Michigan hospitals between January 1982 and September 1985. Patients were identified by reviewing the final diagnoses recorded in the emergency department logs and by a computerized search for the registration numbers of all patients with ICD-9 code 969.0 who were admitted to the hospital. First, the records of all patients with the diagnosis of "drug overdose," "tricyclic overdose," or overdose with a specifically named antidepressant were obtained. To be included in the study, the patient's record needed specific documentation that the ingestion had been witnessed or that there were measurable drug levels of TCAs in blood or urine specimens. From the chart, we estimated the amount of TCA ingested, the time from ingestion to arrival at the hospital, the presence of major clinical signs or symptoms (hypotension, coma, seizure, respiratory depression, dysrhythmia, or death) as well as late complications and ultimate outcome. The QRS duration on the initial ECG was also recorded to determine if it could predict the occurrence of seizures or ventricular dysrhythmias. Initial therapy such as gastric emptying, charcoal administration, and use of cathartics was also determined. Finally, the data were used to test the clinical algorithm in Figure 1.

The algorithm is identical to the one proposed by 


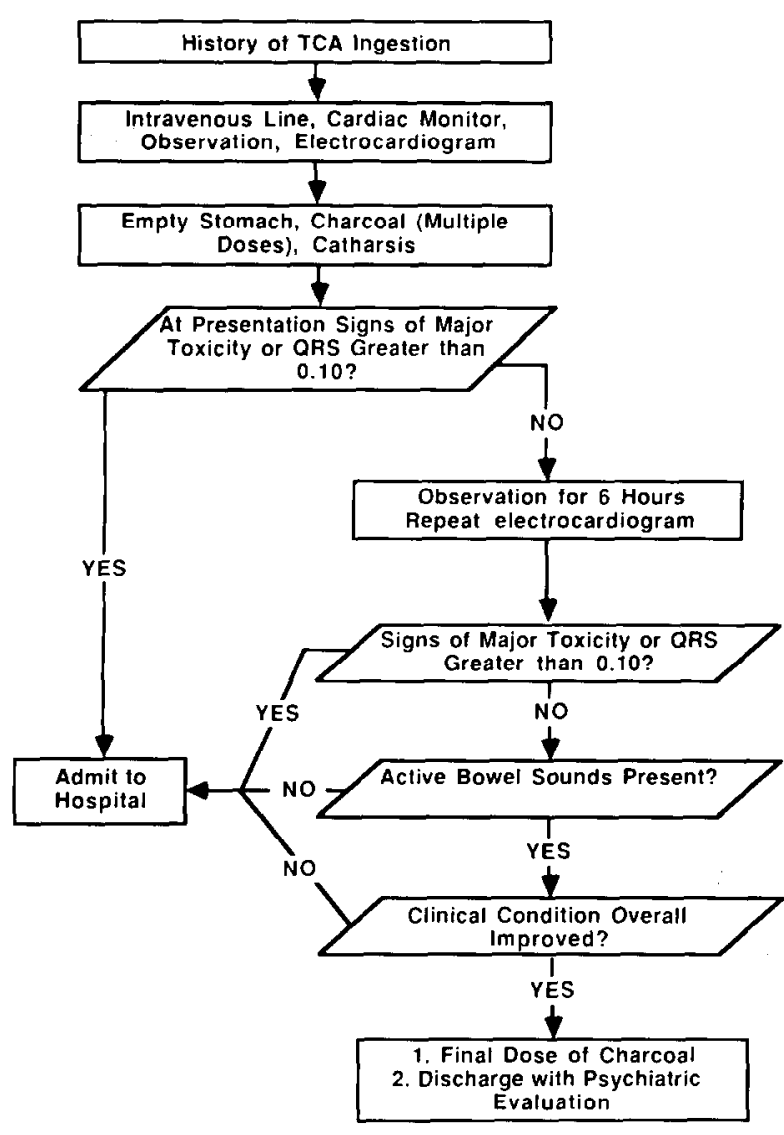

Figure 1. Algorithm for managing patients with tricyclic antidepressant overdosage. Adapted from Callaham. ${ }^{7}$

Callaham, ${ }^{7}$ with one exception. The qualitative term "conduction blocks," has been replaced by "QRS > $0.10 "$ - a more specific term used by Boehnert and Lovejoy. Although there was no specific documentation about bowel sounds other than at the time of adinission in most patients, we assumed that patients who had no symptoms and developed no complications would have had normal bowel sounds at six hours.

The method of Hanley and Lippman-Hand was used to calculate $95 \%$ confidence intervals around the observed rate of complications. When zero complications are observed, the $95 \%$ confidence intervals equal $3 / N$, where $N$ equals the number of cases in the study. ${ }^{9}$

\section{RESULTS}

Of the 48 patients identified as TCA overdoses, $45(93 \%)$ had complete records that could be used to validate the algorithm. The majority $(60 \%)$ of our patients were women and $68 \%$ of patients were be- tween the ages of 18 and 40 . A total of $58 \%$ of patients ingested other agents (most often alcohol and benzodiazapines) in addition to TCAs; the remaining $42 \%$ of patients ingested only TCAs. Estimated time from ingestion to presentation also varied widely, although $60 \%$ were evaluated less than six hours after ingestion. The TCAs ingested by our patients included amitriptyline, nortriptyline, imipramine, desipramine, and doxepin. The estimated amounts of ingested TCAs also varied widely, but it was estimated that one half of patients had ingested $\geq 1,000 \mathrm{mg}$ of the various TCAs. Of the 45 patients reviewed, 44 were admitted to the intensive care unit. Only one patient was discharged from the emergency department (ED); this patient presented without symptoms $20 \mathrm{~h}$ after ingestion of $300 \mathrm{mg}$ of doxepin (only twice the recommended therapeutic daily dose). The presenting signs and symptoms of all patients are shown in Table 1.

All patients had cardiac monitoring and a 12-lead ECG in the ED. Initial therapy with gastric emptying (by ipecac or lavage), activated charcoal, and catharsis was documented in $\mathbf{3 2}$ of $\mathbf{4 8}$ patients. Ten patients received ipecac and charcoal (no documentation of catharsis), and two patients received only ipecac (no documentation of charcoal or catharsis). There was no documentation of gastric emptying, charcoal administration, or catharsis in one patient.

\section{COMPLICATIONS}

Of the 17 patients who presented with major signs or symptoms, 14 developed no further complications. In the remaining three patients, one developed seizures, one developed respiratory depression that required ventilatory support for approximately $48 \mathrm{~h}$, and one developed aspiration pneumonia that required prolonged hospitalization.

In our population, no complications developed after the first $24 \mathrm{~h}$ of hospitalization. All of the patients had constant cardiac monitoring; none developed malignant ventricular dysrhythmias. All patients were ultimately discharged alive and well after receiving appropriate psychiatric care.

\section{ELECTROCARDIOGRAMS}

We also evaluated how the results of ECG alone would predict the development of seizures and ventricular dysrhythmias. Twenty-eight patients had a QRS duration less than $0.10 \mathrm{~s}$; none of these patients developed seizure activity or ventricular dysrhyth- 
Table 1. Signs and Symptoms at Time of Presentation to the ED

\begin{tabular}{lc}
\hline Signs or Symptoms & $\begin{array}{c}\text { Number of } \\
\text { Patients }\end{array}$ \\
\hline None & 28 \\
Serious toxicity & 17 \\
Coma & 12 \\
Seizure & 1 \\
Respiratory depression & 8 \\
Systolic blood pressure $(<90 \mathrm{~mm} \mathrm{Hg})$ & 4 \\
Dysrhythmia & 0 \\
\hline
\end{tabular}

"Since some of the patients had multiple complications, the sum of the individual signs and symptoms is greater than 17 .

mias. Seventeen patients had a QRS duration between 0.10 and $0.16 \mathrm{~s}$; two of these patients had seizure activity but none experienced ventricular dysrhythmias. No patients had a QRS duration greater than $0.16 \mathrm{~s}$.

\section{VALIDATION OF ALGORITHM}

We next attempted to validate the modified algorithm shown in Figure 1. Of the 45 patients, $25(55 \%)$ would have been admitted immediately or within six hours of presentation because they developed major signs or symptoms of TCA poisoning or had a prolonged QRS interval. This group included 17 patients with major signs at the time of presentation, as shown in Table 1. One patient presented without major signs or symptoms but developed coma and respiratory depression within six hours of presentation. Seven patients were without major signs or symptoms but had an initial QRS duration of greater than $0.10 \mathrm{~s}$. Twenty patients $(45 \%)$ would have been discharged from the ED after six hours of observation (assuming normal bowel sounds were present). None of these 20 asymptomatic patients had complications during their hospital courses. The $95 \%$ confidence interval around this observation is 0 to $15 \%$.

\section{DISCUSSION}

Patients who ingest overdoses of TCAs are customarily admitted to the hospital since even patients who are initially asymptomatic may develop complications at a later time. Previous work has shown that serum TCA levels do not correlate with ultimate outcome or prognosis. ${ }^{2.8}$ QRS duration of greater than $0.10 \mathrm{~s}$ in combination with TCA blood levels has been utilized in the past to predict the "seriousness" of TCA overdose; however, it is only recently that the value of the ECG has been further clarified. ${ }^{8}$
Despite these shortcomings, admission criteria for patients with suspected or reported TCA overdose have been proposed. In 1982 Callaham advocated sixhour management of TCA overdoses in the emergency department to identify complicated versus uncomplicated TCA overdose $\mathrm{e}^{10}$; in 1985 he published a clinical algorithm by which these patients could be evaluated. Six hours were felt to be an appropriate length of observation since all reported patients who died from TCA overdose developed major signs of toxicity within two hours of presentation to the ED. ${ }^{7.11}$ Although Callaham's algorithm seems sensible and clinically useful, no studies that test the validity of the algorithm have been reported. Our study determined applicability and validity of Callaham's algorithm to another patient population and also modified the algorithm by including specific criteria for a prolonged QRS interval.

One of the major principles in the algorithm is the use of six hours of observation to identify patients at risk for complications. This concept appeared to be useful in our patients. Seventeen patients had evidence of major TCA toxicity on arrival to the ED; one of them developed further complications within six hours of arrival. Similarly, of the 28 patients who presented without major signs, the only patient who developed life-threatening complications did so within six hours of presentation. Although there are many case reports of patients who have developed late complications of TCA overdose, these complications seem to occur in patients who have obvious toxicity at presentation or who have been given inadequate therapy in the ED. ${ }^{11}$ Almost all (42/45) of our patients had documentation of initial therapy - gastric emptying and administration of activated charcoal. ${ }^{12}$ All patients in our study who experienced complications attributable to TCA overdose did so within six hours. Therefore our data supports the concept that late complications do not occur in properly treated patients who do not have obvious early evidence of toxicity.

The inclusion of specific criteria for a prolonged QRS duration deserves comment. Seven patients who otherwise had no evidence of toxicity had a QRS duration of greater than $0.1 \mathrm{~s}$. None of these patients developed seizures or dysrhythmias. However, previous studies of larger numbers of patients do suggest that these patients, especially those with a QRS duration greater than $0.16 \mathrm{~s}$, are at risk of complications. ${ }^{8}$ Since we had no patients with such a prolonged QRS, we are unable to comment on the validity of that predictor of complications. We should also note that application of the QRS criteria without considering the clinical status of the patient is inap- 
propriate. Some of the patients with evidence of toxicity did not have prolonged QRS intervals in their initial ECG.

It is important to define the limitations of this retrospective and relatively small study. Retrospective studies inherently have missing data, either of clinical information or of follow-up. As suggested by others, ${ }^{13}$ missing information may be less of a problem for studies of patients admitted to intensive care units. We had adequate information on 45 of the 48 records and since $44 / 45$ were admitted to intensive care units, we are confident that we identified major complications. The one piece of information that was missing in most of the records was the presence or absence of bowel sounds at six hours. However, the assumption that bowel sounds were present in the uncomplicated ingestions does not affect our conclusions, since none of these patients developed complications.

A more important limitation is the number of pa- tients in our study. The algorithm identified 20 patients who were at low risk for complications, and none of them developed complications. The $95 \%$ confidence intervals around this observation are relatively wide, 0 to $15 \%$. Larger studies should be performed to obtain narrower confidence intervals that estimate the safety of using the algorithm to manage patients with TCA overdose.

The common practice of admitting nearly all patients to intensive care units is based on rare case reports of late complications developing in asymptomatic paticnts. Our study and others suggest that late complications do not develop in patients who are given adequate therapy and who have no initial evidence of toxicity. Further work needs to be done to evaluate safe strategies for managing patients with TCA overdose. We conclude that the modified algorithm is clinically reasonable and is suitable for testing with large numbers of patients in a prospective fashion.

\section{REFERENCES}

1. Greenland P, Howe TA: Cardiac monitoring in tricyclic antidepressant overdose. Heart Lung 1981; 10:856-859.

2. Braithewaite RA, Crome P, Dawling S: Amitriptyline overdosage: plasma concentrations and clinical features. Br J Clin Pharmacol 1979; 8:388-389.

3. Spiker DG, Weiss AN, Chang SS, et al: Tricyclic antidepressant overdose: Clinical presentation and plasma levels. Clin Pharmacol Ther 1975; 18:539-546.

4. Rosen MJ: Tricyclic antidepressant overdose: Clinical features and management. Heart Lung 1983; 12:222-225.

5. Langou RA, Van Dyke C, Tahan SR, et al: Cardiovascular manifestations of tricyclic antidepressant overdose. Heart Lung 1980; 100:458-464.

6. Goldberg RJ, Capone RJ, Hunt JD: Cardiac complications following tricyclic antidepressant overdose. JAMA 1985; 254:1772-1775.

7. Callaham M, Kassel D: Epidemiology of fatal tricyclic antidepressant ingestion: implications for management. Ann Emerg Med 1985; 14:29-37.
8. Boehnert MT, Lovejoy FH: Value of the QRS duration vs the serum drug level in predicting seizures and ventricular arrythmias after an acute overdose of tricyclic antidepressants. $N$ Engl J Med 1985; 313:474-479.

9. Hanley JA, Lippman-Hand A: If nothing goes wrong, is everything all right? Interpreting zero numberators. JAMA 1983; 249:1743-1745.

10. Callaham M: Admission criteria for tricyclic antidepressant ingestion. West J Med 1982; 137:425-429.

11. Pentel P, Olson KR, Becker CE, et al: Late complications of tricyclic antidepressant overdose. West J Med 1983; 138:423424.

12. Swartz CM, Sherman A: The treatment of tricyclic antidepressant overdose with repeated charcoal. J Clin Psychopharmacol 1984; 4:336-340.

13. Singer DE, Mulley AG (letter): Prognosis after hospitalization for suspected myocardial infarction. $N$ Engl $J$ Med 1980; $303: 1365$. 